\title{
CMS4SI Structural Identification Approach for Interpreting Measurements
}

\author{
James-A. Goulet \\ PhD. Student, EPFL \\ Swiss Fed. Inst. of Tech. \\ Lausanne, Switzerland \\ James.Goulet@epfl.ch
}

James-A. Goulet, born 1984, received his civil engineering and M. Sc. degree from the Univ. of Laval, Canada.

\section{Summary}

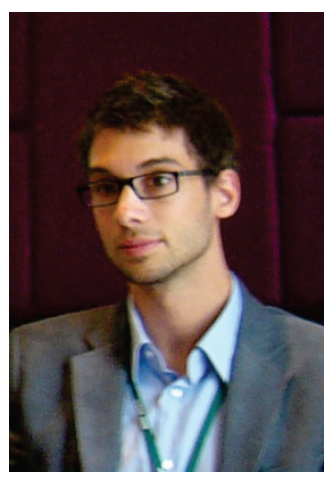

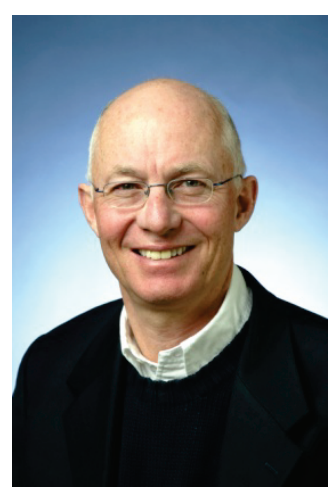

The ageing of existing structures and new innovative designs are increasing the necessity for a greater understanding of structural behaviour. A better understanding would improve effectiveness of activities such as assessing reserve capacity, evaluating load increases and replacement decision making. Identification methodologies are needed to indicate the right behaviour using indirect measurements and behaviour models.

This paper proposes a methodology that is able to accommodate multiple explanations while overcoming limitations of other SI approaches. The algorithm is called Candidate Model Search for System Identification (CMS4SI). Metrology guidelines are extended for use in the field of system identification while systematically including uncertainties and their correlations. The CMS4SI approach provides the necessary robustness and simplicity to support decisions related to the identification and understanding of structural behaviour. The approach is evaluated by full scaletesting of the Langensand Bridge. A critical aspect for meaningful identification is the uncertainty associated with model simplifications. The adaptation of clustering techniques and the use of radar plots allow for a convenient visualisation of results involving several parameters. Finally, models that are identified can be used to perform predictions of unmeasured behaviour, thereby supporting infrastructure management.

Keywords: CMS4SI, Structural identification (SI), Performance assessment, Uncertainty, Correlation, Radar plot

\section{Introduction}

Finding models that correctly predict structural behaviour is not feasible using advanced models alone. Even when using a refined, task-adapted numerical model, there are too many unknowns. Without structural measurements, values for parameters such as material properties, interface conditions (ex: composite interaction, connection stiffness, etc...), and cannot be assessed. Resources are always limited, and therefore sensors cannot be placed everywhere in every direction to measure all important phenomena on civil structures. Therefore, identification methodologies are needed to indicate the right behaviour using indirect measurements and behaviour models.

Model-based system identification (SI) is an active research area in many engineering fields. Several approaches of varying complexity are available.

\subsection{Multiple-Model Methods}

Multiple-model approaches have been introduced by Raphael and Smith [2]. The starting point for the method was to be compatible with the presence of errors in inverse engineering tasks. Therefore, more than one model should be retained for the evaluation of observed behaviour. Over the years, the concept evolved to propose sampling through model composition [3, 4] based on stochastic search [5].

Previous work has proposed thresholds to represent uncertainties that separate potential and rejected models. However, uncertainty combination was not developed into a robust and systematic 
methodology. A first approach [6] combines the uncertainties by calculating a total variance through summing the variance from different sources. Such process may lead to inaccurate estimates of uncertainties in the case where variables are not independent. The second approach [1] was the calculation of a total standard deviation obtained by summing the standard deviation from several sources. This gives an upper bound corresponding to the case where the uncertainties are perfectly and positively correlated with each other. These two approaches are particular cases of the general law of propagation of uncertainties, see Joint Committee for Guides in Metrology (JCGM) [7] For the purposes of interpretation, advanced techniques were proposed by Saitta et al [8-10] to find

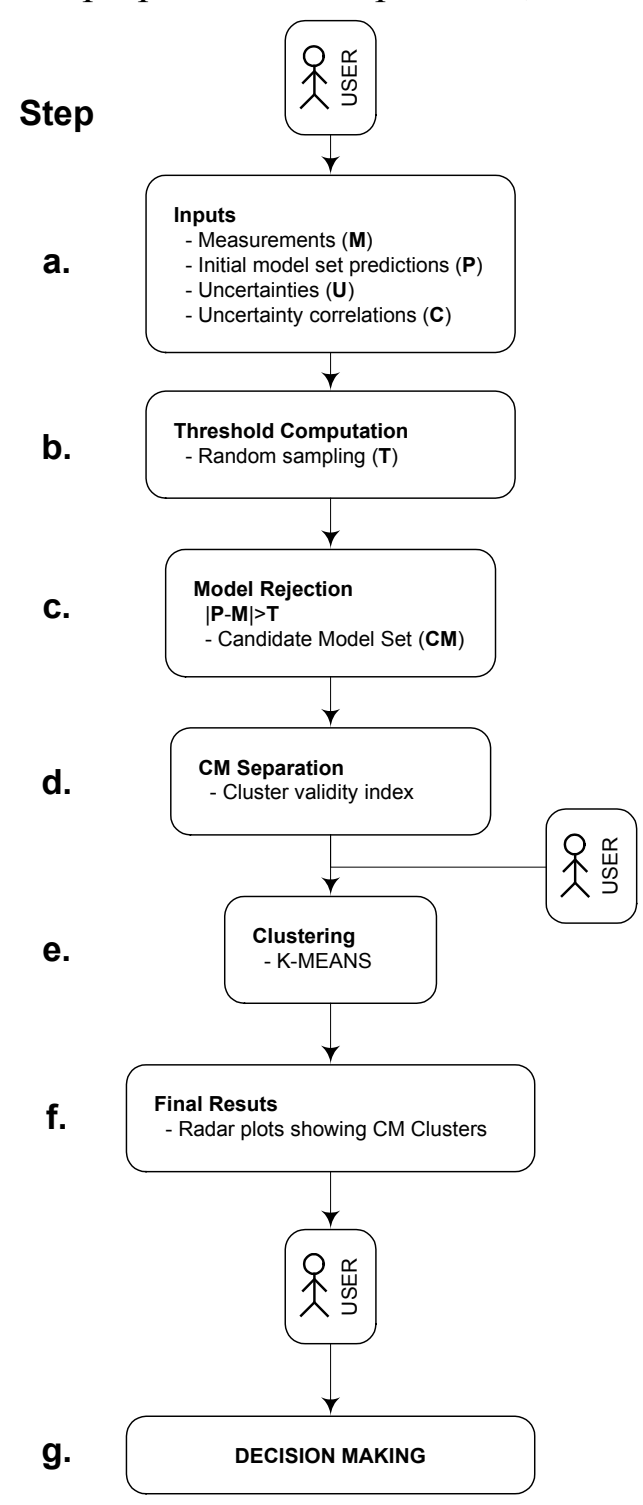

Figure 1-CMS4SI Framework clusters within the candidate model set. An additional approach proposed by Smith and Saitta [11] used parameter reduction through principal component analysis in order to improve visualisation and interpretation of results that often lie in multi-dimensional spaces. However, in this case, even if the visualisation is facilitated, results lose physical sense and this makes the task of interpreting them harder. Although multiple-model approaches may resolve some of the shortcomings of residual minimisation and traditional probabilistic methods, they require a methodology that separates potential models from an initial sampled set while including uncertainties from both modelling and measurements along with their correlations. JCGM [7, 12, 13] already proposed a part of the solution. However, it has never been extended to be applicable to system identification.

This paper proposes a multiple-model methodology that is able to accommodate multiple explanations while overcoming limitations of other SI approaches. The methodology is called Candidate Model Search for System Identification (CMS4SI). In this approach, metrology guidelines are extended for use in the field of system identification. Its applicability is demonstrated on a fullscale bridge in Section 3.

\section{CMS4SI}

The Candidate Model Search for System Identification (CMS4SI) methodology helps identify the behaviour of a system. In this case, the systems of interest are civil structures. Figure 1 presents a flowchart of the principal steps associated with the identification process. The fundamental principle behind the method is the following:

When uncertainties are adequately evaluated and a right model is present in the initial model set, this model should be included in the candidate model set 95 times out of 100 .

Therefore, rather than finding plausible models, the approach aims to discard models for which predictions are sufficiently different from measurements so that the right model is retained in the candidate model set at least ninety-five percent of the time. Each step presented in Figure 1 is detailed in following sections.

\subsection{Measurements and Initial Model Set (Step a.)}

The method starts with measurements coming from static-load tests (the approach may also be extended to dynamic measurements). Each measurement consists of a reading (or an average of multiple readings) of a given sensor for a specific load case. The same sensor may provide more than one measurement if there is more than one load case.

The second input consists of the initial model set (IMS). The IMS contains model predictions 
coming from evaluations of a general finite-element template model that is solved for many sets of parameters (model instances). The goal is to find the possible values for free parameters according to the measured behaviour. Each parameter set is generated using Latin Hypercube Sampling (LHS) [14]. It assumes that good solutions may be found anywhere in the model space. LHS draws homogeneously distributed samples in order to maximise sampling efficiency. A probability density function (PDF) is assigned for each free parameter of the model. Each is defined according to where plausible solutions are expected.

The number of model instances in the IMS should be as high as possible in order to ensure that the right model is present in the set. For structural identification tasks similar to the bridge discussed in this paper, this number lies between 5'000 and 25'000 model instances in order to obtain results using inexpensive computing resources. For each instance, predictions are obtained where measurements are carried out.

\subsection{Uncertainties (Step a.)}

Uncertainties are the primary inputs of the method. Evaluation of uncertainties is required for both modelling and measuring processes.

Each uncertainty source is evaluated using either a statistical or a Bayesian approach [7]. Regardless of the evaluation method, the Gaussian distribution is employed for most uncertainty estimations. However, for some uncertainties, such as those related to model simplification, this distribution is inadequate. Another distribution to represent lack of knowledge is a uniform distribution where the upper and lower bounds are fixed using user judgement (Bayesian approach). Since the real value for an error may never be known exactly, the bounds of a uniform distribution also have an uncertainty related to their positions. One may assume that the uncertainty on the position of the bounds is a fraction $(\beta)$ of the upstream uncertainty. Pursuing that reasoning, the uncertainty on the bounds position also has uncertainty which is once again a fraction of the upstream uncertainty. If the process is continued an infinite number of times, the convolution of these distributions leads to the Extended Uniform Distribution (EUD) representing in a systematic manner, our perception of some uncertainties (for example the modelling uncertainties).

\subsection{Uncertainty Correlations (Step a.)}

Combining uncertainty in order to obtain the maximal plausible error (threshold value) also requires the qualification of the correlation between uncertainties. There are two types of correlations.

The first is the correlation between uncertainty sources for either a given measured or predicted quantity. The method is able to account for the correlation between several uncertainty sources associated with either the same measurement or the same prediction. However, for common civil engineering cases the uncertainty sources mentioned in the previous section are often all independent. For example, the correlation between uncertainty on model simplifications is independent from the uncertainty on truck weight.

The second correlation type to account for is the dependencies for a given uncertainty source over either several measured or predicted quantities. These dependencies are evaluated separately for each uncertainty source using either a statistical or a Bayesian approach. For model dependent uncertainties (model fixed parameter values, temperature effects, applied loading, etc.) the correlation is implicitly accounted for during the computation. Therefore, these correlations are not required as a direct input from the user. Further details are given in the next section.

For model independent uncertainty sources, in most cases, only the Bayesian approach can be used to quantify correlations. The correlation values are reported in a table describing the dependency either between each prediction or measurement types (displacement, rotation or strains) for each uncertainty source. Correlation inputs are used by the algorithm to build the complete correlation matrix for model simplification and FEM uncertainties. Since this matrix is symmetric, only half may be provided.

In previous studies such as those described in introduction, uncertainties are often assumed to be independent for each measurement location (often termed to be degrees of freedom (DOF)). However, this assumption is seldom true, especially for structures. For example, consider a single span beam loaded by a concentrated force. If this structure is approximated using FEM, there would 
be a finite number of beam elements and degrees of freedom. Such example is illustrated in Figure 3 for the cases of independent and correlated errors. This structure is made of 9 beam elements and 17 DOF. Its displacement behaviour is used for illustration purposes.

The error between the real and the predicted vertical displacement is $\mathrm{e}_{1}$ for the third node (starting from the left), $e_{2}$ for the fifth node and $e_{3}$ for the sixth node. In most practical cases, these errors are neither perfectly correlated (Figure 2a) nor independent (Figure 2b). They are instead a combination of these two where in most situations, the systematic character is dominant. The errors at the three locations (DOF) are highly correlated. Therefore for such a structure, assuming that the model uncertainties are aleatory variables that are independent for each DOF would not be an adequate representation of the true system.



Independent errors (aleatory)

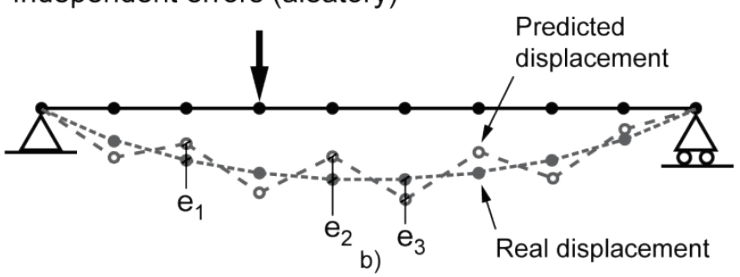

\section{Figure 2 - Error correlation}

Most probabilistic approaches are based on assumptions of independence and randomness. As suggested by JCGM [7], knowing only that the absolute value of these correlations are neither equal to 0 nor 1 , a Bayesian approach can be use to quantify them for each uncertainty source in the presence of each other uncertainty.

\subsection{Threshold Computation (Step b.)}

The threshold represents the maximal plausible error committed during the identification process. This value is computed through stochastic sampling over the uncertainty sources. By first considering only the uncertainties depending on the template model, random samples are taken from each PDF while free model parameters are kept to their mean initial values. The finite element method is used to obtain predictions for the different set of uncertainties. Each evaluation gives a particular response under specific uncertain conditions. This process needs to be repeated approximately $10^{4} /(1-\mathrm{P})$, where $\mathrm{P}$ is the desired coverage in percentage of the whole PDF (coverage interval). For a coverage interval of $95 \%$ this corresponds to $200^{\prime} 000$ samples. In practical terms, this is more than the 1'000 to $25^{\prime} 000$ that it is currently possible to solve using inexpensive computing resources. Use of a small number of samples is inevitably less reliable than using a large sample set. However, this often has a marginal influence since the uncertainties depending on the template model are, for most practical cases, less important than other uncertainty sources described below.

The second step involves drawing random samples from the remaining uncertainty PDFs (Template-model independent such as sensor resolution or additional uncertainties) and adding them to randomly selected prediction set obtained through the previous step. In this second step, it is possible to take several million samples $(>50 \mathrm{M})$ since it does not involve FEM evaluations. The output is a reliable representation of the combined distribution of every uncertainty source occurring in the identification process.

According to the fundamental principle, when the identification uses a single measurement, it has a $5 \%(\mathrm{~F}=1-\mathrm{P}=1-0.95)$ chance of wrongly rejecting the right model. If two measurements are used (this could be either two sensors and one load case or one sensor and two load cases), each of them have a $5 \%$ chance of wrongly rejecting the right model. However, by definition, a model instance is rejected if any measurement falsifies it. Therefore, if the uncertainties over the two measurements and predictions are not perfectly and positively correlated, filtering with two measurements could lead to a probability of rejection of the right model higher than $5 \%$. As a result, when more than one measurement is used, the threshold coverage interval needs to be adjusted in order to ensure that the right model is included in the CM set with a $95 \%$ probability.

For a situation where measurements and prediction uncertainty correlations have absolute values between 0 and 1 , an iterative algorithm is used in order to ensure that the fundamental principle mentioned in the first paragraph of Section 2 is respected. Therefore, the algorithm recursively adds small increments to the coverage interval used to compute the threshold and tests the validity of the 
fundamental principle by generating simulated measurements. This is done by adding uncertainties coming from modelling and measurement to the predictions from a randomly chosen model instance. This process results in simulated measurement for which the right model is known. Several simulated measurements $\left(>10^{\prime} 000\right)$ are used to test if the right model is found in the CM set $95 \%$ of the time. The outcome is a threshold which consistently accommodates for uncertainties and correlations.

\subsection{Model Rejection (Step c.)}

The criterion defining the rejection is a simple expression. For a given model instance, if the difference between a predicted and measured value is larger than the threshold (i.e. the maximal probable error), it is considered to be an unacceptable explanation of structural behaviour. Results retained by this algorithm are grouped into the candidate model set (CM) where each individual is equally likely to be a right representation of the real system.

\subsection{Separation and Clustering (Steps d. and e.)}

Consider the example where an initial set of model instances contains $25^{\prime} 000$ individuals and after having completed the rejection process, $99 \%$ of these models are rejected. In this case, 250 candidate models need to be evaluated. This task becomes overwhelming as the size of the candidate model set increases. To overcome this challenge, clustering techniques adapted by Saitta [8-10] are used. A performance index ranks from 0 to 1 the validity of clusters according to their number. A plot is provided to present the computed validity associated with each number of clusters. Users choose the number of clusters according to their judgement. The algorithm uses this selected number of cluster to separate the CM set using k-means algorithm [15].

In the current context, and contrary to its initial goal, the clustering algorithm is not used to find well defined clusters within the data set but instead, to separate models into families in order to facilitate $\mathrm{CM}$ visualisation. The goal is to separate the $\mathrm{CM}$ set into a manageable number of candidate-model subsets.

\subsection{Visualisation and Decision Making (Steps f. and g.)}

When the system has up to three unknown parameters, it is possible to plot the sub-group of candidate models on a single Cartesian graph. However, the number of graphs required and the complexity of their interpretation increases exponentially with the number of parameters. For example, a four parameter identification would require the interpretation of 12 two-dimensional graphs. For five parameters this number increases to 60. For civil engineering tasks, most applications have more than three parameters.

Radar plots enable comparison of thousands of different models having a large number of parameters. In such graphs, each axis represents a parameter where the intersection takes the respective minimal possible value, and the free end the maximal one. This allows for visual comparisons of clusters and model instances in a multi-dimensional space using a single 2D graph.

This enables the engineer to make decisions that are enhanced by measurements. For example, possible model explaining the measured behaviour while considering uncertainties may be reviewed and used for further visual inspection or non destructive test investigations (NDT) on the structure. Additionally, predictions on unmeasured behaviour may be performed. For example, predictions on the in-service displacement reserve capacity may be evaluated by simulating a given loading on each CM. The upper and lower predicted responses may be used to define the bounds within which the real structure behaviour should lie. Therefore, this approach may be used for predictive applications. The results obtained through the CMS4SI methodology supports decisions such as

- $\quad$ Assessing displacement or fatigue reserve capacity

- $\quad$ Retrofitting evaluations

- $\quad$ Load increases evaluations

- $\quad$ Replacement avoidance

- $\quad$ Visual inspection and NDT investigation planning 


\section{Case-Study: Langensand Bridge}

The example used for static-load tests is the Langensand Bridge built in Lucerne (Switzerland). This structure was under construction (half of width launched) when tested. Therefore, only one half of it is in the scope of the study. This bridge is approximately $80 \mathrm{~m}$ long and has a slender profile (>1:30) see Figure 3a).



Figure 3 - a) Langensand bridge elevation representation [1] b) Langensand cross section [1]

\subsection{Structure Description}

The shaded area in Figure 3b) represents the part of the bridge in place during load testing. It consists of a poured concrete deck on a steel girder. The central part of the bridge is used as roadway and the external parts are sidewalks.

The measurement system used during the identification is composed of six displacement, two rotation and three strain measurements recorded for five load cases. Complete information regarding loading, sensor layout and details are presented in [16].

The template model has four variables which have to be identified: steel girder Young's modulus (E-STEEL), concrete Young's modulus (E-CONC), road surface Young's modulus (E-RS) and the stiffness of the horizontal support created by the bearing devices (U-STIFF). The IMS composed of 5'000 model instances. Each model is analysed using five load-cases. This leads to a total of 25'000

Table 1 - Langensand Bridge Uncertainties

\begin{tabular}{|c|c|c|c|c|c|c|c|c|c|}
\hline Uncertainty source PDF & \multicolumn{3}{|c|}{$\begin{array}{l}\text { Displacement } \\
\text { unit min max }\end{array}$} & \multicolumn{3}{|c|}{$\begin{array}{l}\text { Rotation } \\
\text { unit min ma }\end{array}$} & \multicolumn{3}{|c|}{$\begin{array}{c}\text { Strains } \\
\mathrm{x} \text { unit } \min \max \end{array}$} \\
\hline Sensor resolution Uniform I & $\mathrm{mm}$ & -0.1 & 0.1 & $\mu \mathrm{rad}$ & -0.4 & 0.4 & $\mu \varepsilon$ & -2 & 2 \\
\hline Model simplification \& FEM EUD & $\%$ & 0 & 7 & $\%$ & 0 & 7 & $\%$ & 0 & 15 \\
\hline Mesh refinement EUD & $\%$ & -1 & 0 & $\%$ & -1 & 0 & $\%$ & -2 & 0 \\
\hline Cable losses Uniform & $\%$ & 0 & 0 & $\%$ & 0 & 0 & $\%$ & 0 & 0 \\
\hline \multirow[t]{2}{*}{ Additional uncertainties } & $\%$ & -1 & 1 & $\%$ & -1 & 1 & $\%$ & -1 & 1 \\
\hline & & unit & & & $\min$ & & & $\max$ & \\
\hline \multirow[t]{2}{*}{ Temperature variation Uniform } & & ${ }^{\circ} \mathrm{C}$ & & & 0 & & & 5 & \\
\hline & & unit & & & Mean & & & STD & \\
\hline$\Delta v$ concrete Normal & & - & & & 0 & & & 0.025 & \\
\hline Truck weight Normal & & Ton & & & 35 & & & 0.125 & \\
\hline$\Delta$ t steel plates Normal & & $\%$ & & & 0 & & & 1 & \\
\hline$\Delta t$ pavement Normal & & $\%$ & & & 0 & & & 5 & \\
\hline$\Delta \mathrm{t}$ concrete Normal & & $\%$ & & & 0 & & & 2.5 & \\
\hline Strain sensor positioning Normal & & $\mathrm{mm}$ & & & 0 & & & 5 & \\
\hline Measurement repeatability Normal & & $\mathrm{m} / \mathrm{rad} /$ & $d / \mu \varepsilon$ & & 0 & & $\begin{array}{r}\text { Mea } \\
\text { de }\end{array}$ & $\begin{array}{l}\text { asuren } \\
\text { apende }\end{array}$ & $\begin{array}{l}\text { nent } \\
\text { ent }\end{array}$ \\
\hline
\end{tabular}

\subsection{Uncertainties and Correlations}

Uncertainties related to the identification are described in Table 1. Model simplification, mesh refinement and additional uncertainties are represented as unbounded uniform distributions. For these PDFs, the $\beta$ (see Section 2) parameter is taken to be 0.3 . These values represent the authors perception of the minimal and maximal bounds within which the true error should lie.

Uncertainty dependencies between measurements and between predictions are presented in Table 2 for various uncertainty sources. In this table, titles represent either the prediction or measurement type for which the correlation is defined. Sensor resolution uncertainties which are not mentioned in this Table are taken to be independent for each measurement. As illustrated in Section 2, the error correlation between different degrees of freedom (displacements and rotations) is likely to be highly correlated. The uncertainty correlation between each load path is taken as $95 \%$ of the correlation between a same load-case. This is based on the principle that if an error is present when simulating using a given load-case, it is likely that it is also present when using a different one. The partial correlation matrix provided for load-cases and for each model-independent uncertainty source is used to build the complete correlation matrices describing the relationship between each prediction location. The process described above for predictions is also

applicable for correlation between measurements. These dependencies are used to generate random correlated uncertainties in order to compute the threshold value. 


\subsection{Results}

From the initial set of 5'000 model instances, 2'587 remain as potential candidate models reducing Table 2 - Langensand bridge partial uncertainty correlations matrix

\begin{tabular}{l|ccc|l}
\hline Prediction type & Disp. & Rotation & Strains & Uncertainty source \\
\cline { 2 - 5 } Disp. & 0.9 & - & - & \multirow{2}{*}{ Nodel simplification \& FEM } \\
Rotation & 0.8 & 0.9 & - & Model \\
Strains & 0.7 & 0.7 & 0.8 & \\
\hline Disp. & 0.9 & - & - & \\
Rotation & 0.8 & 0.9 & - & Mesh refinement \\
Strains & 0.7 & 0.7 & 0.8 & \\
\hline Disp. & 0.5 & - & - & \\
Rotation & 0.5 & 0.5 & - & Add. Uncertainties \\
Strains & 0.5 & 0.5 & 0.5 & \\
\hline Measurement type & Disp. & Rotation & Strains & Uncertainty source \\
\hline Displ. & 0.9 & - & - & \\
Rotation & 0.8 & 0.9 & - & Meas. Repeatability \\
Strains & 0.7 & 0.7 & 0.8 & \\
\hline
\end{tabular}

the model space by almost $50 \%$. For this case, the method efficiency at rejecting candidate models is much less than for the previous example which provided a reduction of $88 \%$. This result may be attributed to the uncertainties, notably those associated with model simplifications.

In order to subdivide the model space, the cluster validity index indicates no improvement in cluster validity when the space is divided into more than four clusters.

The four clusters are presented in Figure 2. Each represents possible combinations of parameters which explain measured data while including uncertainties and correlations. The possible solution space helps identifying parameters needing further study in order to reduce uncertainty and improve identification.

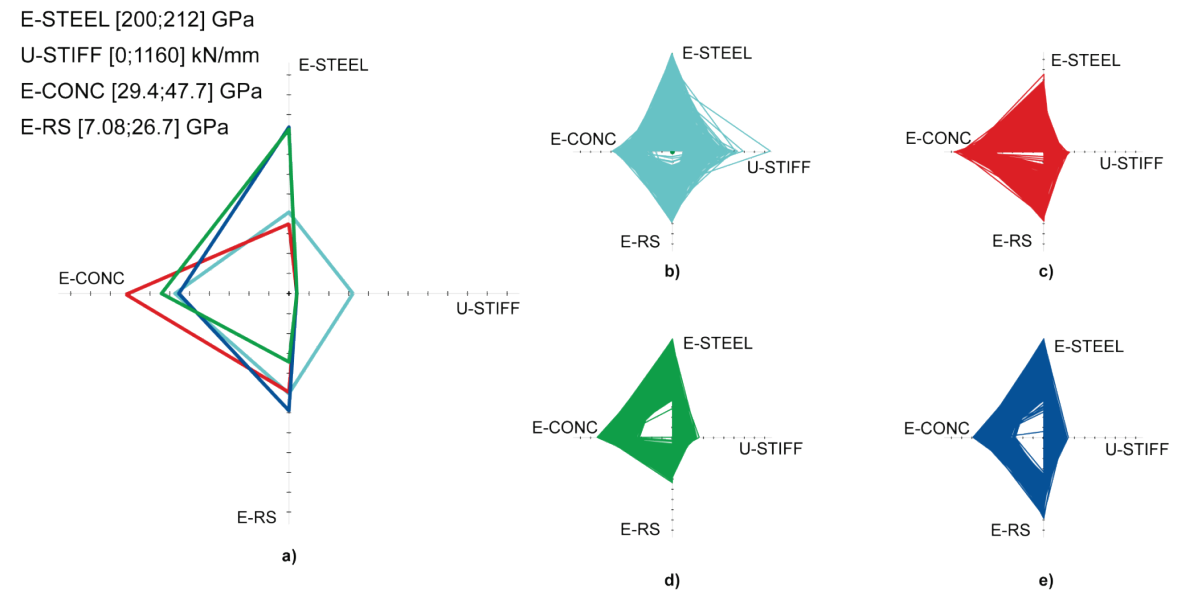

Figure 2- Candidate models. a) Cluster average, b) Cluster \#1, c) Cluster \#2, d) Cluster \#3, e) Cluster \#4
In this case, the cluster b) indicates that the measured behaviour may be explained by a longitudinal displacement restriction of the intended free end of the structure (U-STIFF). Such results can flag anomalous behaviour and justify further on-site visual inspections. As reported by Goulet et al. [1], a blocked support was found on the structure and its stiffness value was evaluated to be approximately $300 \mathrm{kN} / \mathrm{mm}$. Clusters c), d), and e) indicate that range ranges

of possible values are observed for the three remaining parameters (E-STEEL, E-CONC and E-RS). Their exact values cannot be found using the current measurements only. Results can be used, for example, to justify further non-destructive tests in order to reduce such variability. Quantifying steel Young's modulus using non-destructive test to would allow reducing the variability associated with parameter compensation as shown in cluster a).

\subsection{Discussion}

Structural identification is limited often by the complexity of the structure as well as the placement and type of sensors. Uncertainties are present and they may be the limiting factor governing identification. This application shows that the simplifications related to the model may, for full scale structures, be the dominant uncertainty source. Two aspects have to be developed in order to improve structural identification. New methods are required in order to define whether or not measuring a structure is useful. Optimal testing configurations (sensor location and load paths) should be selected using a method that focuses on reducing the candidate model set in the most efficient way. Developments of these aspects are currently the subject of further research.

\section{Conclusions}

This paper proposes a methodology that is able to accommodate multiple explanations while 
overcoming limitations of other SI approaches. The methodology is called Candidate Model Search for System Identification (CMS4SI). Established metrology guidelines are extended for use in the field of system identification while consistently accounting for uncertainties and their correlations.

The CMS4SI approach provides the necessary robustness and simplicity to support decisions related to the identification and understanding of structural behaviour. The validity of the approach is demonstrated through a simple test case and its applicability is tested with full scale test performed on the Langensand Bridge.

The importance of errors is underlined by this last example. In many cases, uncertainties are the limiting factor for identification capability. Uncertainties may be too large to uniquely identify the behaviour of a structure. A critical aspect that determines the number of candidate models is the uncertainty associated with model simplifications.

The adaptation of clustering techniques and the use of radar plots allow for a convenient visualisation of results involving several parameters.

\section{Acknowledgements}

This research is funded by the Swiss National Science Foundation under contract no. 200020117670/1. The authors want to thanks Dr. Prakash Kripakaran for his contribution to this paper.

\section{References}

[1] Goulet, J.-A., P. Kripakaran, and I.F.C. Smith, Multi-Model Structural Performance Monitoring. Journal of Structural Engineering, Submitted for publication (In press).

[2] Raphael, B. and I. Smith, Finding the right model for bridge diagnosis, in Artificial Intelligence in Structural Engineering. 1998. p. 308-319.

[3] Robert-Nicoud, Y., et al., Model identification of bridges using measurement data. Computer-Aided Civil and Infrastructure Engineering, 2005. 20(2): p. 118-131.

[4] Robert-Nicoud, Y., B. Raphael, and I.F.C. Smith, System Identification through Model Composition and Stochastic Search. Journal of Computing in Civil Engineering, 2005. 19(3): p. 239-247.

[5] Raphael, B. and I.F.C. Smith, A direct stochastic algorithm for global search. Applied Mathematics and Computation, 2003. 146(2-3): p. 729-758.

[6] Ravindran, S., P. Kripakaran, and I.F.C. Smith, Evaluation reliability of multiple-model system identification, in 14th EG-ICE Workshop. 2007: Maribor, Slovenia.

[7] JCGM, Evaluation of measurement data - Guide to the expression of uncertainty in measurement. 2008, Bureau international des poids et mesures: Paris, France.

[8] Saitta, S., B. Raphael, and I.F.C. Smith, Data mining techniques for improving the reliability of system identification. Advanced Engineering Informatics, 2005. 19(4): p. 289-298.

[9] Saitta, S., B. Raphael, and I.F.C. Smith, Combining two data mining methods for system identification, in Intelligent Computing in Engineering and Architecture. 2006, Springer Berlin: Berlin. p. 606-614.

[10] Saitta, S., B. Raphael, and I.F.C. Smith, A bounded index for cluster validity, in Machine Learning and Data Mining in Pattern Recognition, Proceedings, P. Perner, Editor. 2007, Springer Berlin: Berlin. p. 174-187.

[11] Smith, I.F.C. and S. Saitta, Improving knowledge of structural system behavior through multiple models. Journal of Structural Engineering, 2008. 134(4): p. 553-561.

[12] JCGM, ed. Evaluation of measurement data - Supplement 1 to the "Guide to the expression of uncertainty in measurement" - Propagation of distributions using a Monte Carlo method. 2008: Paris, France.

[13] JCGM, ed. International vocabulary of metrology - Basic and general concepts and associated terms (VIM). 2008: Paris, France.

[14] McKay, M.D., R.J. Beckman, and W.J. Conover, A Comparison of Three Methods for Selecting Values of Input Variables in the Analysis of Output from a Computer Code. Technometrics, 1979. 21(2): p. 239-245.

[15] MacQueen, J. Some methods for classification and analysis of multivariate observations. in Fifth Berkeley Symp. on Math. Statist. and Prob. 1967: Univ. of Calif. Press, 1967.

[16] Goulet, J.-A., P. Kripakaran, and I.F.C. Smith, Multi-Model Structural Performance Monitoring. Journal of Structural Engineering, Submitted for publication (Under revision process). 